\title{
Acute Acquired Comitant Esotropia: Etiology, Clinical Course, and Management
}

\author{
Apatsa Lekskul (iD \\ Nichaboon Chotkajornkiat \\ Wadakarn Wuthisiri \\ Phantaraporn Tangtammaruk (D) \\ Department of Ophthalmology, \\ Ramathibodi Hospital, Mahidol \\ University, Bangkok, Thailand
}

Purpose: To identify the etiologies, clinical course and management of acute acquired comitant esotropia in Ramathibodi Hospital, Thailand.

Methods: Thirty patients who were diagnosed with acute acquired comitant esotropia at Ramathibodi Hospital from January 12017 to December 312019 were identified using electronic medical records, from which demographic, etiology, clinical course and management, laboratory, and neuroimaging data were collected.

Results: The etiologies of acute acquired comitant esotropia were Swan (16.67\%), BurianFranceschetti (30.00\%), Bielschowsky (36.67\%), Arnold Chiari malformation (3.33\%) and decompensated esophoria (13.33\%). Mean age of onset was $19.8 \pm 18.3$ years. Mean angle of esodeviation was $28.4 \pm 12.1$ prism diopters for distance fixation and $29.3 \pm 11.8$ prism diopters for near fixation. Refraction differed between age groups: children under 10 years had mild hyperopia (median +0.63 diopters, first quartile +0.25 diopters, third quartile +0.75 diopters) and teenagers (10-18 years old) had emmetropia to mild myopia (median +0.25 diopters, first quartile -2.50 diopters, third quartile +0.75 diopters), whereas adults had mild to moderate myopia (median -0.75 diopters, first quartile -5.25 diopters, third quartile \pm 0.00 diopters). Twelve patients $(40.00 \%)$ were prescribed spectacles and surgical intervention was performed in 26 patients (86.67\%). All patients except one case of Arnold Chiari malformation $(96.67 \%)$ maintained normal binocular function and alignment following strabismus surgery or spectacles correction.

Conclusion: Bielschowsky was the most common etiology of acute acquired comitant esotropia in our study. We suggest that refraction should be performed in all patients with acute acquired comitant esotropia. Most etiologies were benign and might not require neuroimaging. However, neuroimaging is recommended in those with atypical presentations, such as nystagmus, headache, or cerebellar signs. Surgical intervention with a $0.5-1.0 \mathrm{~mm}$ increase in recession was effective for restoring ocular alignment and binocular function in our patients.

Keywords: AACE, acute acquired comitant esotropia, strabismus, acquired esotropia, etiologies

\section{Introduction}

Acute acquired comitant esotropia (AACE) is described as a presentation of strabismus and is characterized by a sudden comitant esotropia with diplopia after infancy. ${ }^{1}$ AACE is a rare disease that occurs in around $0.3 \%$ of children with strabismus. ${ }^{2,3}$ AACE has been divided into the three categories by Burian and Miller: ${ }^{4}$ Swan (type 1), which has a sudden onset due to interrupted fusion by monocular occlusion or vision loss; Burian-Franceschetti (type 2), which may be
Correspondence: Phantaraporn Tangtammaruk

270 Rama 6 Road, Thung Phayathai

Subdistrict, Ratchathewi District,

Bangkok, 10400, Thailand

$\mathrm{Tel}+66611947887$

Email pp.phantaraporn@gmail.com 
caused by physical or psychological stress and is demonstrated by large deviations, small refractive errors, and minimal accommodation; and Bielschowsky (type 3), which is associated with moderated myopia or might result from excessive near work activities. ${ }^{5}$

Etiologies of AACE have been reported previously, ${ }^{1-4}$ such as refractive error; decompensated esophoria with progressive intermittent, horizontal, or binocular diplopia that dramatically converts into constant comitant large angle esotropia; or history of near work activities. These etiologies are benign or have functional causes. However, many studies have reported intracranial diseases, such as elevated intracranial pressure, brain glioma, Arnold Chiari malformation, and thalamic or cerebellar tumors as etiologies, which require neuroimaging examinations, especially in older patients. ${ }^{2,5-8}$

Spontaneous recovery of AACE is unusual. Numerous reports have suggested that treatment of AACE may include spectacles correction, botulinum toxin, or surgical intervention for good prognosis of binocular function. ${ }^{9-11}$

The aim of our study was to examine the etiology, clinical course, and management of AACE at Ramathibodi Hospital. Moreover, we also aimed to study the refractive stage and outcomes following treatment in several age groups of AACE patients.

\section{Materials and Methods}

We conducted a retrospective study of patients with AACE who were diagnosed from January 12017 to December 31 2019 at Ramathibodi Hospital, which is a tertiary referral center in Thailand. The study protocol was approved by the Human Research Ethics Committee Faculty of Medicine Ramathibodi Hospital, Mahidol University and adhered to the tenets of the Health Insurance Portability and Accountability Act and the Declaration of Helsinki. Written informed consent was obtained from all participants and their guardians for their clinical records to be used in this study.

\section{Patients}

The diagnosis of AACE was based on an acute onset of comitant esotropia with a deviation difference of $<5$ prism diopters (PD) in all gaze directions, photographic evidence of the absence of strabismus before esotropia onset, and normal gaze movement and diplopia.

Exclusion criteria included history of ocular surgery (except for refractive surgery), paralysis, ocular trauma, accommodative spasm and accommodative strabismus (hyperopia $\geq+2.00$ diopters, D) with resolution of deviation with full hyperopic correction.
Medical history and demographic data, which included age, gender, and onset of diplopia were collected from electronic medical records. Duration more than 5 hours per day of near work activities including smartphone usage and reading books prior to the time of onset was recorded. Orthoptic examinations, which included ocular movement and deviation were measured using an alternate prism cover test performed with near $(33 \mathrm{~cm})$ and distance fixations $(6 \mathrm{~m})$. Refractive errors and best-corrected visual acuity (BCVA) were measured in all cases. Cycloplegic refraction was recorded by administering $1 \%$ cyclopentolate eye drops twice every 10 minutes for 20 minutes in all patients and a third drop was administered 10 minutes after the second drop if the pupil size was less than $6 \mathrm{~mm}$. Refractive error was measured using standard retinoscopy. The spherical equivalent (SE) was calculated as the sum of the dioptric power of the sphere and half of the cylinder. Myopia was defined as SE of more myopic than $-0.50 \mathrm{D}$ and hyperopia was defined as SE of more hyperopia than +0.50 D. Binocular function was assessed using the Worth's Four Dot Test or a synoptophore. Stereoacuity was measured using the Titmus test card at $40 \mathrm{~cm}$ and the circle test of the Randot stereopsis test.

Patients who had neurological symptoms or recurrence of AACE underwent computed tomography or magnetic resonance imaging (MRI). ${ }^{12}$

The treatments received by patients (ie, spectacles or surgical intervention) were recorded and patients were followed up 6 months later. Deviation and binocular function were reassessed during the follow-up period ( 2 weeks to 6 months).

\section{Statistical Analysis}

Continuous variables are presented as means \pm standard deviations (SD). Continuous non-normal distributions are presented as median, quartile $1(\mathrm{Q} 1)$, quartile $3(\mathrm{Q} 3)$. As the data showed a non-normal distribution, we used one-way analysis of variance. Statistical analyses were performed using the SPSS software (version 23 for Windows). For all tests, $p<0.05$ was considered significant.

\section{Results}

During the 3-year study period, 30 patients were diagnosed with AACE at Ramathibodi Hospital. The various etiologies of AACE are presented in Table 1, Figure 1 and included type 1: Swan (16.67\%), type 2: BurianFranceschetti (30.00\%), type 3: Bielschowsky (36.67\%), 
Table I Etiologies, Deviation and Binocularity of AACE Patients

\begin{tabular}{|c|c|c|c|c|c|c|}
\hline Etiology of AACE & $\mathbf{N}=\mathbf{3 0}$ & Percentages & $\begin{array}{l}\text { Deviation Prior } \\
\text { to Treatment } \\
\text { (Prism Diopters) }\end{array}$ & $\begin{array}{l}\text { Deviation Post } \\
\text { Treatment } \\
\text { (Prism } \\
\text { Diopters) }\end{array}$ & $\begin{array}{l}\text { Binocularity } \\
\text { Post } \\
\text { Treatment }\end{array}$ & $\begin{array}{l}\text { Stereoacuity Post } \\
\text { Treatment (Range, } \\
\text { Arc Seconds) }\end{array}$ \\
\hline Swan type & 5 & 16.67 & $33.5 \pm 7.0$ & $5.0 \pm 3.5$ & Fusion & $40-60$ \\
\hline Franceschetti type & 9 & 30.00 & $31.0 \pm 9.1$ & $3.6 \pm 3.1$ & Fusion & $30-60$ \\
\hline Bielschowshy type & 11 & 36.67 & $28.5 \pm 14.5$ & $3.3 \pm 3.5$ & Fusion & $40-60$ \\
\hline Arnold Chiari malformation & I & 3.33 & $32.5 \pm 0.0$ & $4.0 \pm 0.0$ & Suppression & Absence \\
\hline Decompensated esophoria & 4 & 13.33 & $18.3 \pm 8.7$ & $4.0 \pm 3.3$ & Fusion & $30-60$ \\
\hline
\end{tabular}

Arnold Chiari malformation (3.33\%) and decompensated esophoria (13.33\%).

Mean age of AACE onset was $19.8 \pm 18.3$ years and mean age of AACE diagnosis was $22.9 \pm 16.1$ years. Patients with Bielschowsky had a mean onset age of 19.1 \pm 15.8 years. Of all type 3 cases, $81.82 \%$ were associated with myopia $(-3.27 \pm 2.91 \mathrm{D})$. Over half of Bielschowsky patients $(54.55 \%)$ had a history of excessive convergence tone that was associated with near work, such as smartphone use $(83.33 \%)$ and reading activity $(16.67 \%)$.
Mean angle of esodeviations was $28.4 \pm 12.1 \mathrm{PD}$ (range 3-50 PD) for distance fixation and $29.3 \pm 11.8$ PD (range 4-50 PD) for near fixation.

BCVA was 20/20 or better in both eyes in patients aged $>10$ years and $20 / 30$ or better in patients aged $<10$ years. Refractions of each age group of AACE patients are shown in Table 2. Refraction differed between age groups. The two younger groups of AACE patients (aged $<10$ years and 10-18 years) had mild hyperopia (median +0.63 diopters, $\mathrm{Q} 1+0.25$ diopters, $\mathrm{Q} 3+0.75$ diopters)

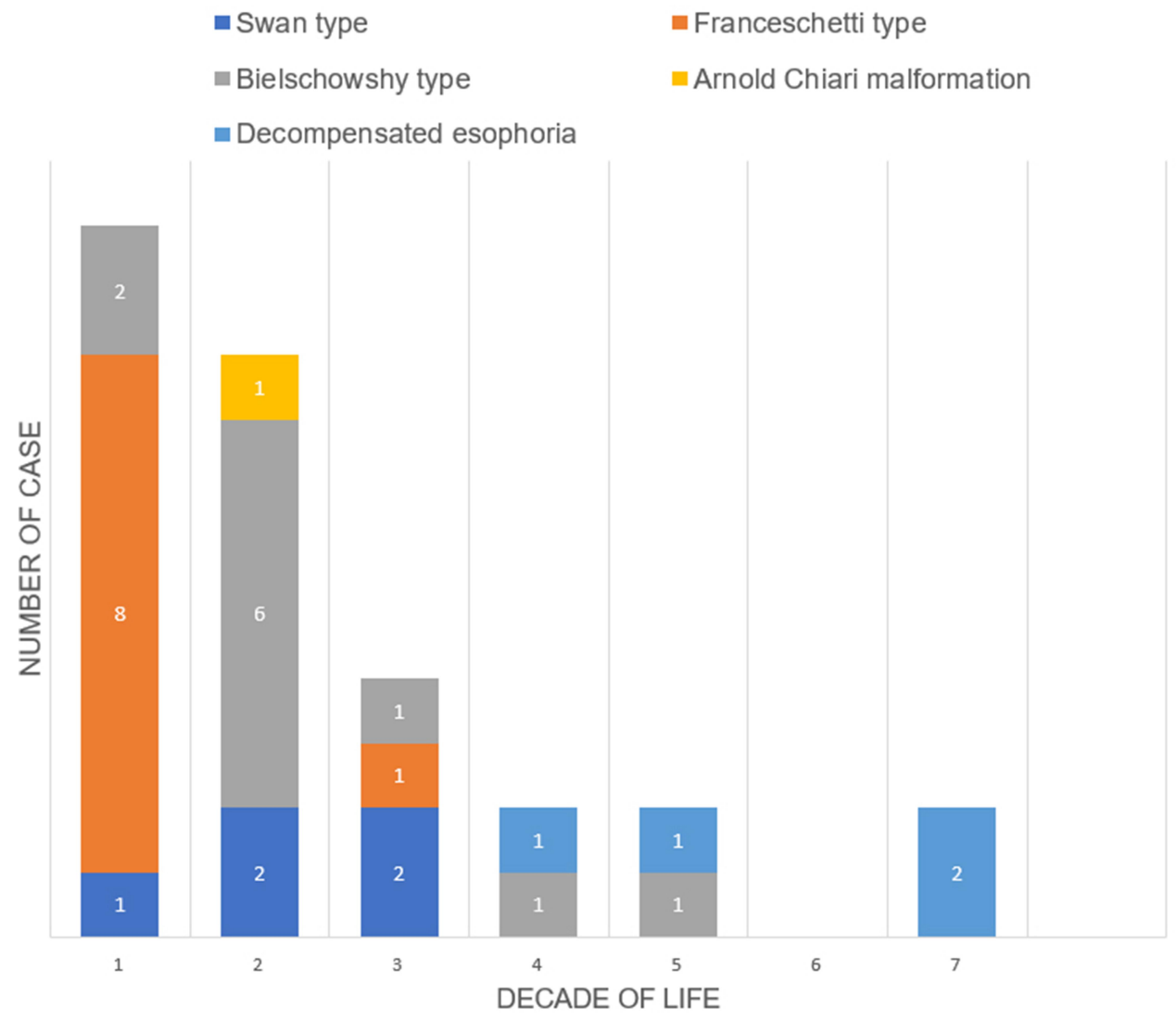

Figure I Frequency and etiology of AACE patients, per decade of life. 
Table 2 The Refraction of AACE Patients Related to Age

\begin{tabular}{|l|r|r|r|}
\hline & Children <10 Years & Teenager 10-18 Years & Adults >18 Years \\
\hline N (patients) & 6 & 9 & 15 \\
SE, median (QI, Q3) & $+0.63(+0.25,+0.75)$ & $+0.25(-2.50,+0.75)$ & $-0.75(-5.25, \pm 0.00)$ \\
\hline
\end{tabular}

Note: Statistical analysis was performed using one-way analysis of variance.

Abbreviations: SE, spherical equivalent; $\mathrm{Q}$, quartile.

and emmetropia to mild myopia (median +0.25 diopters, Q1 -2.50 diopters, $\mathrm{Q} 3+0.75$ diopters), respectively. The adult group ( $>18$ years) displayed higher myopia (median -0.75 diopters, Q1 -5.25 diopters, Q3 \pm 0.00 diopters).

Twelve patients $(40.00 \%)$ were prescribed full correction spectacles, of which eight had partial response, and four had complete response with no esotropia or diplopia. Thirteen patients $(43.33 \%)$ underwent neuroimaging because of history of headache $(38.46 \%)$, recurrence diplopia (23.08\%), nystagmus $(7.69 \%)$, or unreliable history $(38.46 \%)$. Arnold Chiari malformation was found in one patient and all other patients' scans were unremarkable. None of the patients who did not require imaging developed intracranial disease, and the mean follow-up period was 2.1 years (range 1.1-3.0 years).

Twenty-six patients $(86.67 \%)$ received surgical interventions, which included unilateral medial rectus recession (3 cases, 11.54\%), bilateral medial rectus recession (18 cases, $69.23 \%$ ), and unilateral medial rectus recession with lateral rectus resection ( 2 cases, 7.69\%). Three patients (11.54\%) underwent a second operation: unilateral medial rectus recession followed by another medial rectus recession. Final ocular alignment after surgery was within $8 \mathrm{PD}$ in all patients $(3.9 \pm$ 3.9 PD, range 0-8 PD). Figures 2 and 3 show two example cases of AACE.

All patients (96.67\%) except one, who had Arnold Chiari malformation, maintained normal binocular function and good alignment after surgery or spectacles correction.

\section{Discussion}

The most common etiology of AACE in our study was Bielschowsky, followed by Burian-Franceschetti, Swan, and decompensated AACE, which is consistent with the findings of Buch and Vinding. ${ }^{2}$ Another less common etiologies of AACE was Arnold Chiari malformation.

Previous studies ${ }^{13,14}$ have reported that the onset of AACE usually occurs during infancy or early childhood. This is markedly different from our findings, where the mean age of AACE onset was $19.8 \pm 18.3$ years. The older age of onset observed in our study may explain why the Bielschowsky type was the most common etiology of AACE; this type is caused by prolonged uncorrected myopia and excessive near work in adults, which results in an imbalance of the convergence and divergence tone of the extraocular muscle and an increased force of the medial rectus muscles, leading to esotropia. ${ }^{15-17}$ Moreover, our findings support the hypothesis that in Asian populations, AACE is more common in older children and adults than in younger children ${ }^{15}$ for the same aforementioned reason.

Interestingly, our study is the first study to analyze refractive error data in AACE patients. We found that the refraction of AACE patients differed between age groups. Adults had higher myopia compared with younger children aged $<10$ years. These findings are in line with a previous study ${ }^{15}$ that demonstrated children under 10 years had mild
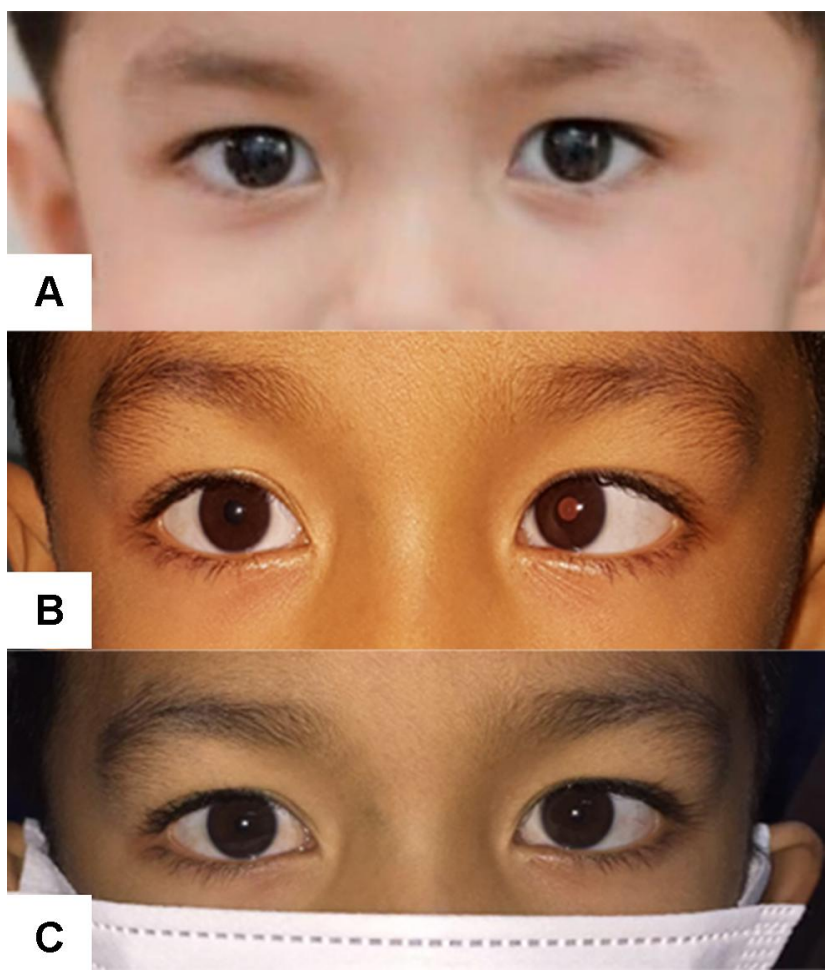

Figure 2 Example case I of AACE: (A) photo prior to onset, (B) photo of deviation prior to treatment and (C) photo after surgical intervention. 


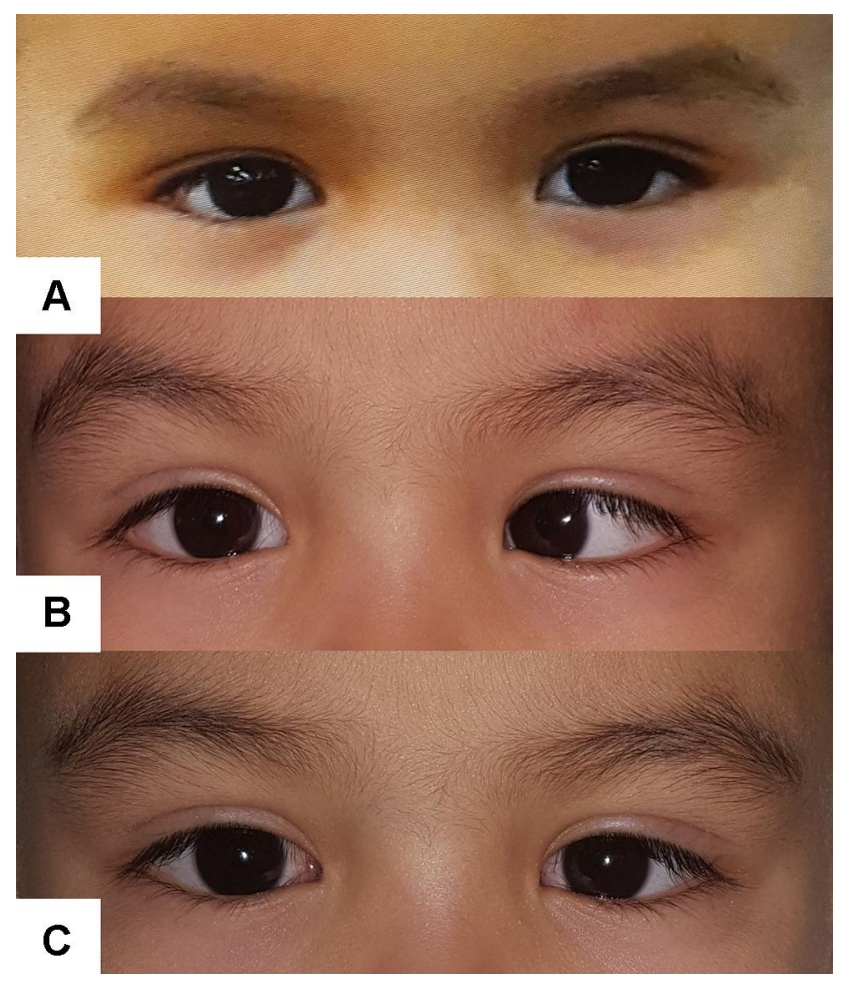

Figure 3 Example case 2 of AACE: (A) photo prior to onset, (B) photo of deviation prior to treatment and $(\mathbf{C})$ photo after surgical intervention.

hyperopia and children aged 10-18 years had mild myopia, whereas adults had moderate to high myopia. Twelve patients with refractive error were prescribed spectacles. Four patients who responded completely to spectacle correction had mild myopia $(-2.25 \pm 2.03 \mathrm{D})$, whereas eight patients who responded partially to spectacle correction had moderate myopia $(-5.86 \pm 2.59 \mathrm{D})$ and required surgical intervention following spectacles correction.

Goldman and Nelson ${ }^{19}$ recommend that for AACE cases who have a dramatic onset of diplopia with large comitant esodeviations, medical history should be thoroughly reviewed for cyclic esodeviation, divergence insufficiency, paretic strabismus, and myasthenia gravis. Moreover, complete ophthalmic and neurological examinations are recommended in patients with a suspected neurological condition. In our study, 13 patients $(43.33 \%)$ who had neurological signs and symptoms, such as headache, nystagmus, recurrence of AACE, and incomplete history underwent neuroimaging. We reported only one case (7.69\%) of Arnold Chiari malformation. The patient was an 11-year-old girl who was first diagnosed at age 10, with an esodeviation of 35 and 30 PD for distance and near fixations, respectively, and gaze-evoked nystagmus. After surgical correction, the patient gained normal alignment but without recovery of binocular function. The decision of when neuroimaging should be performed remains controversial. Previous studies ${ }^{6-8}$ have reported incidences of up to $10 \%$ for neurological diseases that are associated with AACE. Isolated AACE is usually a benign condition but it can also be the first sign of a serious neurological disease. However, there is no single clinical symptom or sign that indicates the presence of a neurological disease. We recommend neuroimaging in patients who have unclear precipitating history or abnormal ocular and neurological symptoms, such as headache, cerebellar imbalance, weakness, or nystagmus.

In our study, the mean angle of esodeviations was 28.4 \pm 12.1 PD (range, 3-50 PD) for distance fixation and 29.3 \pm 12.1 PD (range, 4-50 PD) for near fixation. Most patients $(86.67 \%)$ underwent surgical intervention, which successfully resolved diplopia. Postoperative angle deviation in all patients was within 8 PD. A second operation was performed in three cases because of recurrence of esotropia after the first operation. We augmented the amount of recession on Parks, ${ }^{20}$ standard surgical number by $0.5-1.0 \mathrm{~mm}$, which resulted in successful restoration of binocular function in all patients without intracranial disease, as was demonstrated in a previous study. ${ }^{9-11,18}$ Cai et $\mathrm{al}^{18}$ reported that the distance from the insertion of the medial rectus to the limbus was shorter $(4.8 \pm 0.4 \mathrm{~mm})$ in Chinese AACE patients due to strengthened convergence tonus and subsequent esotropia. In a general Thai population, Athavale et $\mathrm{al}^{21}$ reported that the distance of the insertion of the medial rectus to the limbus was $5.7 \mathrm{~mm}$, which is similar to the general Chinese population (5.4 \pm $0.4 \mathrm{~mm}) .{ }^{18}$ Based on these anatomical findings in AACE patients, we increased the amount of recession by $0.5-1.0 \mathrm{~mm}$, which resulted in good outcomes in our AACE patients.

Our study has some limitations. First, we were only able to study 30 patients in 3 years because AACE is less common than infantile esotropia and we only acquired data from a single center. Second, because of the retrospective study design, medical history data were limited. Future prospective studies of AACE patients are recommended.

In conclusion, Bielschowsky was the most common etiology in our patients. Our findings recommend that ophthalmologists perform refraction in all cases who present with AACE. Although neuroimaging in AACE remains controversial, our study revealed that it is necessary in patients with atypical presentations, such as nystagmus, 
headache, or cerebellar signs. Surgical intervention with augmented recession that was $0.5-1.0 \mathrm{~mm}$ larger than standard operations enabled successful restoration of ocular alignment and binocular function.

\section{Informed Consent}

Informed consent was obtained from all individual participants included in the study. Ethical approval was waived by the Human Research Ethics Committee Faculty of Medicine Ramathibodi Hospital, Mahidol University and adhered to the tenets of the Health Insurance Portability and Accountability Act and the Declaration of Helsinki in view of the retrospective nature of the study and all the procedures being performed were part of the routine care.

\section{Acknowledgments}

We thank Sarina Iwabuchi, PhD, from Edanz Group (https://en-author-services.edanz.com/ac) for editing a draft of this manuscript.

\section{Disclosure}

The authors report no conflicts of interest in this work.

\section{References}

1. Clark AC, Nelson LB, Simon JW, Wagner R, Rubin SE. Acute acquired comitant esotropia. Br J Ophthalmol. 1989;73(8):636-638. doi:10.1136/bjo.73.8.636

2. Buch H, Vinding T. Acute acquired comitant esotropia of childhood: a classification based on 48 children. Acta Ophthalmol. 2015;93 (6):568-574. doi:10.1111/aos.12730

3. Neena R, Giridhar A. Acute acquired comitant esotropia in children: a benign entity or an ominous sign? Kerala J Ophthalmol. 2019;31 (1):33-38. doi:10.4103/kjo.kjo_11_19

4. Burian HM, Miller JE. Comitant convergent strabismus with acute onset. Am J Ophthalmol. 1958;45(4):55-64. doi:10.1016/00029394(58)90223-X

5. Lee HS, Park SW, Heo H. Acute acquired comitant esotropia related to excessive smartphone use. BMC Ophthalmol. 2016;16(1):37. doi:10.1186/s12886-016-0213-5

Clinical Ophthalmology

\section{Publish your work in this journal}

Clinical Ophthalmology is an international, peer-reviewed journal covering all subspecialties within ophthalmology. Key topics include: Optometry; Visual science; Pharmacology and drug therapy in eye diseases; Basic Sciences; Primary and Secondary eye care; Patient Safety and Quality of Care Improvements. This journal is indexed on PubMed

Submit your manuscript here: https://www.dovepress.com/clinical-ophthalmology-journal
6. Hoyt CS, Good WV. Acute onset concomitant esotropia: when is it a sign of serious neurological disease? Br J Ophthalmol. 1995;79 (5):498-501. doi:10.1136/bjo.79.5.498

7. Akman A, Dayanir V, Sanaç AS, Kansu T. Acquired esotropia as presenting sign of cranio-cervical junction anomalies. NeuroOphthalmology. 1995;15(6):311-314. doi:10.3109/ 01658109509044620

8. Lyons CJ, Tiffin PAC, Oystreck D. Acute acquired comitant esotropia: a prospective study. Eye. 1999;13(5):617-620. doi:10.1038/ eye.1999.169

9. Spierer A. Acute concomitant esotropia of adulthood. Ophthalmology. 2003;110(5):1053-1056. doi:10.1016/S01616420(03)00102-7

10. Kassem RR, Elhilali HM. Factors affecting sensory functions after successful postoperative ocular alignment of acquired esotropia. J AAPOS. 2006;10(2):112-116. doi:10.1016/j.jaapos.2006.01.001

11. Chen J, Deng D, Sun Y, et al. Acute acquired concomitant esotropia: clinical features, classification, and etiology. Medicine. 2015;94(51): e2273. doi:10.1097/MD.0000000000002273

12. Ansons AM, Devis H. Diagnosis and Management of Ocular Motility Disorders. 3rd ed. Oxford: Blackwell Science; 2001.

13. Sturm V, Menke MN, Knecht PB, Schöffler C. Long-term follow-up of children with acute acquired concomitant esotropia. J AAPOS. 2011;15(4):317-320. doi:10.1016/j.jaapos.2011.03.018

14. Savino G, Colucci D, Rebecchi MT, Dickmann A. Acute onset concomitant esotropia: sensorial evaluation, prism adaptation test, and surgery planning. J Pediatr Ophthalmol Strabismus. 2005;42 (6):342-348. doi:10.3928/01913913-20051101-02

15. Fu T, Wang J, Levin M, Xi P, Li D, Li J. Clinical features of acute acquired comitant esotropia in the Chinese populations. Medicine. 2017;96(46):e8528. doi:10.1097/MD.0000000000008528

16. Kemmanu V, Hegde K, Seetharam R, Shetty BK. Varied aetiology of acute acquired comitant esotropia: a case series. Oman J Ophthalmol. 2012;5:103-105. doi:10.4103/0974-620X.99373

17. Mohan K, Dhankar V. Acute concomitant esotropia. J Pediatr Ophthalmol Strabismus. 2002;39(5):304-306. doi:10.3928/01913913-20020901-13

18. Cai C, Dai H, Shen Y. Clinical characteristics and surgical outcomes of acute acquired comitant Esotropia. BMC Ophthalmol. 2019;173.

19. Goldman HD, Nelson LB. Acute acquired comitant esotropia. Ann Ophthalmol. 1985;17(12):777-778.

20. Parks MM. Atlas of Strabismus Surgery. Philadelphia: Harper and Row Publishing; 1983.

21. Athavale S, Kotgirwar S, Lalwani R. Rectus and oblique muscles of eyeball: a morphometric study of Indian population. Anat Cell Biol. 2015;48(3):201-204. doi:10.5115/acb.2015.48.3.201
Central and CAS, and is the official journal of The Society of Clinical Ophthalmology (SCO). The manuscript management system is completely online and includes a very quick and fair peer-review system, which is all easy to use. Visit http://www.dovepress.com/ testimonials.php to read real quotes from published authors. 